\title{
Modeling LGN Responses during Free-Viewing: A Possible Role of Microscopic Eye Movements in the Refinement of Cortical Orientation Selectivity
}

\author{
Michele Rucci, ${ }^{1}$ Gerald M. Edelman, ${ }^{2}$ and Jonathan Wray ${ }^{2}$ \\ ${ }^{1}$ Department of Cognitive and Neural Systems, Boston University, Boston, Massachusetts 02215, and ${ }^{2}$ The \\ Neurosciences Institute, San Diego, California 92121
}

\begin{abstract}
Neural activity appears to be essential for the normal development of the orientation-selective responses of cortical cells. It has been proposed that the correlated activity of LGN cells is a crucial component for shaping the receptive fields of cortical simple cells into adjacent, oriented subregions alternately receiving ON- and OFF-center excitatory geniculate inputs. After eye opening, the spatiotemporal structure of neural activity in the early stages of the visual pathway depends not only on the characteristics of the environment, but also on the way the environment is scanned. In this study, we use computational modeling to investigate how eye movements might affect the refinement of orientation tuning in the presence of a Hebbian scheme of synaptic plasticity. Visual input consisting of natural
\end{abstract}

Since the discovery that high percentages of cells in the primary visual cortex of different mammal species respond preferentially to luminance edges with specific orientations (Hubel and Wiesel, 1962), the developmental origin of cortical orientation selectivity has been studied intensively. Substantial experimental evidence (Zahs and Stryker, 1988; Chapman et al., 1991; Reid and Alonso, 1995; Ferster et al., 1996; Chung and Ferster, 1998) supports the proposal that the adjacent oriented excitatory and inhibitory subregions present in the receptive fields of simple cells receive selective input from geniculate $\mathrm{ON}$ - and OFF-center cells in the same retinotopic positions (Hubel and Wiesel, 1962). However, the mechanisms underlying the emergence of such segregation of thalamic afferents are still unclear.

The essential elements of cortical orientation selectivity seem to develop before the exposure to patterned visual input (Wiesel and Hubel, 1974; Blakemore and Van Sluyters, 1975; Sherk and Stryker, 1976; Fregnac and Imbert, 1978; Albus and Wolf, 1984; Chapman and Stryker, 1993; Chapman et al., 1996; Crair et al., 1998). Nevertheless, visual experience appears essential for both refining orientation selectivity and maintaining the normal response properties of cortical neurons (Pettigrew, 1974; Blakemore and Van Sluyters, 1975; Buisseret and Imbert, 1976; Fregnac and Imbert, 1978; Hirsch, 1985; Chapman and Stryker, 1993; Crair et al., 1998). A number of experimental findings (Stryker and Har-

Received Nov. 2, 1999; revised March 29, 2000; accepted March 31, 2000.

This work was carried out as part of the theoretical neurobiology program at The Neurosciences Institute, which is supported by the Neurosciences Research Foundation. We thank the Fellows of The Neurosciences Institute, in particular Dr. Joe Gally, Dr. Chris Habeck, and Dr. Giulio Tononi, for useful discussions.

Correspondence should be addressed to Dr. Michele Rucci, Boston University, 677 Beacon Street, Boston, MA 02215. E-mail: rucci@cns.bu.edu.

Copyright (C) 2000 Society for Neuroscience $0270-6474 / 00 / 204708-13 \$ 15.00 / 0$ scenes scanned by varying types of eye movements was used to activate a spatiotemporal model of LGN cells. In the presence of different types of movement, significantly different patterns of activity were found in the LGN. Specific patterns of correlation required for the development of segregated cortical receptive field subregions were observed in the case of micromovements, but were not seen in the case of saccades or static presentation of natural visual input. These results suggest an important role for the eye movements occurring during fixation in the refinement of orientation selectivity.

Key words: visual development; microsaccade; natural visual experience; computer model; cat; visual fixation; Hebbian plasticity

ris, 1986; Fregnac et al., 1992; Chapman and Stryker, 1993; Weliki and Katz, 1997) support the hypothesis that the development of orientation-selective responses relies on Hebbian/covariance mechanisms of plasticity, which transform the temporal contiguity of firing patterns into spatial proximity of synaptic contacts (Stent, 1973; Changeux and Danchin, 1976). According to this hypothesis, the clustering and/or segregation of neural inputs emerges from the stabilization of synchronously firing afferents onto common postsynaptic neurons and the destabilization of nonsynchronous ones. A necessary requirement of the Hebbian hypothesis is a consistency between the correlated activity of thalamic afferents and the organization of simple-cell receptive fields. Synchronous activation is required among geniculate cells of the same type (ON- or OFF-center) with receptive fields located at distances smaller than the width of a simple-cell subregion, and among cells of opposite polarity with receptive fields at distances comparable to the separation between adjacent subregions. Modeling studies (Linsker, 1986; Miyashita and Tanaka, 1992; Miller, 1994) have shown the feasibility of this proposal assuming similar spatiotemporal patterns of spontaneous activity in the LGN before eye opening.

After eye opening, the spatiotemporal structure of LGN activity depends not only on the characteristics of the visual input, but also on the movements performed by the animal while exploring its environment. It may be expected that changes in the visual input induced by these movements play an important role in shaping the responses of neurons in the visual system. Experiments in which kittens were raised with their eyes paralyzed have shown basic deficiencies in the development of visually guided behavior (Hein et al., 1979), as well as impairments in ocular dominance plasticity (Freeman and Bonds, 1979; Singer and Raushecker, 1982). In addition, it has been shown that eye move- 
ments are necessary for the reestablishment of cortical orientation selectivity in dark-reared kittens exposed to visual experience within the critical period (Buisseret et al., 1978; Gary-Bobo et al., 1986). This indicates that simultaneous experience of visual input and eye movements (and/or eye movement proprioception) may be necessary for the refinement of orientation selectivity (Buisseret, 1995).

The focus of this paper is on how visual experience and eye movements might jointly influence the refinement of orientation selectivity under the assumption of a Hebbian mechanism of synaptic plasticity. We have analyzed the second order statistical structure of neural activity in a model of cat LGN. In the absence of eye movements, when a natural visual environment was observed statically, similar to the way it is examined by animals with their eyes paralyzed, we found that the simulated responses of geniculate cells of the same type at any separation smaller than the receptive field of a simple cell were strongly correlated. These spatial patterns of covarying geniculate activity did not match the structure of simple-cell receptive fields. A similar result was obtained when natural scenes were scanned through saccades. Conversely, in the case of micromovements, including both microsaccades and the combination of ocular drift and tremor, strong correlations were measured among cells of the same type located nearby and among cells of opposite types at distances compatible with the separation between different subregions in the receptive fields of simple cells. In this case, the covarying activity of LGN units closely matched the spatial structure of simple-cell receptive fields. These findings support a role for micromovements in the refinement of orientation-selective cortical responses.

\section{MATERIALS AND METHODS}

In the experiments described in this paper we simulated the activity of geniculate cells with receptive fields in different positions of the visual field, while receiving visual input in the presence of different types of eye movements. The following describes each element of the model in detail.

\section{Modeling the activity of LGN cells}

LGN cells were modeled as linear elements with quasi-separable spatial and temporal components as proposed by Cai et al. (1997). This model, derived using the reverse-correlation technique, has been shown to produce accurate estimates of the activity of different types of LGN cells. Changes in the instantaneous firing rates with respect to the level of spontaneous activity $l_{\mathrm{xy}}(t)$, were generated by evaluating the spatiotemporal convolution of the input image $I$ with the receptive field kernel $K$ :

$$
\begin{array}{r}
l_{\mathrm{xy}}(t)=[K(x, y, t) \star I(x, y, t)]_{\theta}=\left[\int _ { 0 } ^ { \mathrm { t } } \int _ { - \infty } ^ { \infty } \int _ { - \infty } ^ { \infty } K ( x ^ { \prime } , y ^ { \prime } , t ^ { \prime } ) I \left(x-x^{\prime},\right.\right. \\
\left.\left.y-y^{\prime}, t-t^{\prime}\right) d x^{\prime} d y^{\prime} d t^{\prime}\right]_{\theta},
\end{array}
$$

where $\star$ is the symbol for convolution, $(x, y)$ and $t$ are the spatial and temporal variables, and the operator $[.]_{\theta}$ indicates rectification $\left([x]_{\theta}=x-\right.$ $\theta$ if $x>\theta, 0$ otherwise). For each cell, the kernel $K$ consisted of two additive components, representing the center $(c)$ and the periphery $(s)$ of the receptive field, respectively. Each of these two contributions was separable in its spatial $(F)$ and temporal $(G)$ elements:

$$
K(x, y, t)=F_{\mathrm{c}}(x, y) G_{\mathrm{c}}(t)-F_{\mathrm{s}}(x, y) G_{\mathrm{s}}(t) .
$$

The spatial receptive fields of both center and surround were modeled as two-dimensional (2D) Gaussians, with a common space constant for both dimensions:

$$
F(x, y)=\left(A / 2 \pi \sigma^{2}\right) \exp \left(-\left(x^{2}+y^{2}\right) / 2 \sigma^{2}\right) .
$$

As is the case for their biological counterparts, ON-center units were characterized by an increase in their activation when a spot of light was flashed at the center of their receptive field and were inhibited when stimulated in the periphery. The opposite was true for OFF-center units, which were equivalent to $\mathrm{ON}$ units with reversed sign in the spatial receptive fields. $F$ was set to zero for distances larger than $3 \sigma$. The spatial parameters, $A_{\mathrm{c}}, A_{\mathrm{s}}, \sigma_{\mathrm{c}}, \sigma_{\mathrm{s}}$, varied with eccentricity following measurements performed by Linsenmeier et al. (1982). Linear interpolation as described in Linsenmeier et al. (1982) was used to estimate the sizes of the 2D Gaussians on the basis of eccentricity, and the amplitudes of excitatory and inhibitory zones on the basis of their respective sizes. The specific values of the parameters used in the simulations are given in Results.

Following Cai et al. (1997), the temporal profile of the response was given by:

$$
\begin{aligned}
& G_{\mathrm{c}}(t)=k_{1} \frac{\left[c_{1}\left(t-t_{1}\right)\right]^{\mathrm{n}_{1}} \exp \left(-c_{1}\left(t-t_{1}\right)\right)}{n_{1}^{\mathrm{n}_{1}} e^{-\mathrm{n}_{1}}} \\
&-k_{2} \frac{\left[c_{2}\left(t-t_{2}\right)\right]^{\mathrm{n}_{2}} \exp \left(-c_{2}\left(t-t_{2}\right)\right)}{n_{2}^{\mathrm{n}_{2}} e^{-\mathrm{n}_{2}}}
\end{aligned}
$$

and

$$
G_{\mathrm{s}}(t)=G_{\mathrm{s}}\left(t-t_{\mathrm{d}}\right)
$$

i.e., the temporal function for the periphery was the same as that for the center, except for the time delay $t_{\mathrm{d}}$. To reduce the intense computational load of the simulations, only nonlagged, ON- and OFF-center X cells were considered, with temporal parameters $k_{1}=1, c_{1}=60 \mathrm{~s}^{-1}, t_{1}=0$ sec, $n_{1}=2, k_{2}=0.6, c_{2}=40 \mathrm{~s}^{-1}, t_{2}=0 \mathrm{sec}, n_{2}=2$. For all units, a time delay $t_{\mathrm{d}}=3 \mathrm{msec}$ was used.

In the simulations described in this paper, unless explicitly stated otherwise, geniculate cells were assumed to operate in their linear range, and rectification was not considered (i.e., $\theta=-\infty$ in Eq. 1). When rectification was present, different thresholds were used for cells with opposite polarities. Rectification thresholds for each image and for cells with different polarities were defined as a fraction $c$ of the negative peaks of steady-state activity (the mean value of steady-state activity was assumed to be zero), and rectification levels as their complementary values $(1-c)$. Thus, a rectification of $50 \%$ indicates that half of the range of steady-state negative responses has been eliminated, whereas only positive responses are present with a rectification of $100 \%$.

\section{Modeling eye movements}

Modeled eye movements included saccades (both large-scale saccades and microsaccades), ocular drift, and tremor.

Saccades. Voluntary saccadic eye movements, the fast shifts of gaze among fixation points, were modeled by assuming a generalized exponential distribution of fixation times (Harris et al., 1988). According to this model, the probability of two saccades following each other by an interval of $t$ msec was given by:

$$
f(t)=\left\{\begin{array}{cc}
(1 / \beta) e^{-(\mathrm{t}-\alpha) / \beta} & t>\alpha \\
0 & t \leq \alpha
\end{array},\right.
$$

where $\alpha$ is a refractory period that prevented the occurrence of new saccadic movements immediately after each saccade. Although this model was originally proposed to describe fixation times in humans, a similar skewed distribution of intersaccadic intervals during free-viewing has also been observed in other species, such as cats and birds (Harris et al., 1988). In the experiments, we set $\alpha=150 \mathrm{msec}$ and $\beta=300 \mathrm{msec}$, to produce an average of roughly two saccades per second. The amplitude and direction of a saccade were randomly selected among all possible saccades that would keep the point of fixation on the image. All possible saccades had an equal probability of being selected. This assumption neglects the fact that saccades may occur toward preferred points in the scene and that the properties of the visual input around these points may bias the measured patterns of correlation. Following data described in the literature (Ditchburn, 1973), the duration $D_{\mathrm{s}}$ of each saccade was proportional to its amplitude $M_{\mathrm{s}}$ :

$$
D_{\mathrm{s}}=v_{s}^{-1}\left(M_{\mathrm{s}}-m_{\mathrm{s}}\right)+d_{\mathrm{s}},
$$

where the velocity $v_{\mathrm{s}}$ was a random variable uniformly distributed between 0.4 and $0.6^{\circ} / \mathrm{msec}, m_{\mathrm{s}}=10^{\circ}$ and $d_{\mathrm{s}}=40 \mathrm{msec}$. A modulation of 
geniculate activity was present in correspondence of each saccade. Neural activity around the time of a saccade was multiplied by the modulatory function:

$$
G(t)=\left\{\begin{array}{cc}
1+A_{\text {pre }}\left(t-T_{\mathrm{e}}\right)^{2} e^{\left(-0.02\left(\mathrm{~T}_{\mathrm{e}}-\mathrm{t}\right)\right)} & t<T_{\mathrm{e}} \\
1+A_{\text {post }}\left(t-T_{\mathrm{e}}\right)^{2} e^{\left(-0.02\left(\mathrm{t}-\mathrm{T}_{\mathrm{e}}\right)\right)} & t \geq T_{\mathrm{e}}
\end{array}\right.
$$

where $T_{\mathrm{e}}$ is the time at which the saccade ends, $A_{\text {pre }}=-7.4 \times 10^{-5}$ and $A_{\text {post }}=1.5 \times 10^{-4}$. In this way the initial suppression of activity with a peak of $10 \%$, gradually reversed to a $20 \%$ facilitation with peak occurring $100 \mathrm{msec}$ after the end of the saccade (Lee and Malpeli (1998)).

Micromovements. Micromovements included microsaccades, ocular drift, and tremor. Microsaccades were modeled in a similar way to voluntary saccades, with amplitude randomly selected from a uniform distribution between 1 and $10 \mathrm{~min}$ of arc [the frequency characteristics of microsaccades can be found in Ditchburn (1973)]. No modulation of LGN activity was present in the case of microsaccades.

Ocular drift and tremor were modeled together following a method similar to the one proposed by Eizenman et al. (1985). According to this model, the power spectrum of ocular drift and tremor can be approximated by two processes: a Poisson process filtered by a second-order eye plant transfer function over the frequency range $0-40 \mathrm{~Hz}$ where the power declines as $1 / f^{2}$, and a cyclo-stationary process that produces a broad spectral peak in the range of $40-100 \mathrm{~Hz}$. The two terms represent the irregular discharge rate of motor units for frequency $<40 \mathrm{~Hz}$ and their more regular firing pattern for higher frequencies, respectively (Kuboki, 1957; Sindermann et al., 1978). For simplicity, given its predominant contribution, only the first term with power spectrum proportional to $1 / f^{2}$ was considered. Parameters were adjusted to give a mean amplitude of $1.21^{\circ}$ and a mean velocity equal to $14.9^{\circ} / \mathrm{sec}$, which are the values measured in the cat (Olivier et al., 1993).

\section{Data collection and analysis}

The activity of 10 simulated LGN units was analyzed when input images were scanned through sequences of eye movements. Unit receptive fields were equispaced along a chosen axis, and the orientation of this axis was changed systematically to sample the full spectrum of possible directions. The results shown in this paper are averages over all examined directions. This allowed a direct comparison between the results of the simulations and neurophysiological data from cortical simple cells (Wilson and Sherman, 1976; Jones and Palmer, 1987b). For most of the experiments described in this paper, the distance between the receptive fields of two adjacent simulated units was equal to 8 arcmin. Thirty images extracted from a database of natural scenes (van Hateren and van der Schaaf, 1998) were used. These images consisted of $1024 \times 1024$ pixels, spanning $24^{\circ}$ of visual angle. The degree of correlation between unit activity was evaluated in the presence of different types of eye movements. In most of the experiments, each image was examined for a period of $250 \mathrm{sec}$, during which eye movements occurred.

Levels of correlations in the activity of LGN units were measured by means of correlation coefficients. Given a unit of type $a$ (ON- or OFFcenter) at position $i$, and one of type $b$ at position $j$, with activation $u_{\mathrm{i}}$ and $u_{\mathrm{j}}$, the correlation of activity was evaluated as:

$$
C_{\mathrm{ij}}^{\mathrm{ab}}=\frac{\left\langle\left(u_{\mathrm{i}}(t)-\bar{u}_{\mathrm{i}}\right)\left(u_{\mathrm{j}}(t)-\bar{u}_{\mathrm{j}}\right)\right\rangle}{\sigma_{\mathrm{i}} \sigma_{\mathrm{j}}}=\frac{c_{\mathrm{ij}}^{\mathrm{ab}}(0)}{\sqrt{c_{\mathrm{ij}}^{\mathrm{aa}}(0) c_{\mathrm{ij}}^{\mathrm{bb}}(0)}},
$$

where $c_{\mathrm{ij}}^{\mathrm{ab}}$ is the cross-covariance of units activity, $c_{\mathrm{ij}}^{\mathrm{ab}}$ and $c_{\mathrm{ij}}^{\mathrm{bb}}$ are their autocovariances, $\bar{u}_{\mathrm{i}}$ and $\sigma_{\mathrm{i}}$ are the mean and variance of the response of the $i$-th LGN cell. The mean values of activity $\left(\bar{u}_{\mathrm{i}}, \bar{u}_{\mathrm{j}}\right)$ were evaluated over a time window of length $T$. This window must not be confused with the duration length of the simulated recordings (usually $5 \mathrm{sec}$, selected on the basis of the available computational power) over which the external average $\langle$.$\rangle was estimated. In most of the experiments described in this$ paper, $\bar{u}_{\mathrm{i}}$ and $\bar{u}_{\mathrm{j}}$ were running averages of neural activity. The alternative possibility of evaluating the actual "covariance" over a window $T$ (in which the duration of the experiment is also set to $T$ ) produced results that were practically indistinguishable from the ones presented in this paper. As expressed by Equation 1, the deviations in the activity of OFF cells with respect to their resting levels were equal in magnitude and opposite in direction to the deviations of the activity of ON cells. As a result, in the linear range of geniculate responses, $C^{\text {ONON }}$ was equal to $C$ OFFOFF. The relative level of correlation between units of the same and different types at positions $i$ and $j$ in the LGN was measured by means of the correlation difference, $C_{\mathrm{ij}}^{\mathrm{D}}=C_{\mathrm{ij}}^{\mathrm{ONON}}-C_{\mathrm{ij}}^{\mathrm{ONOFF}}$, where the two terms are the correlation coefficients evaluated between the two ON units at positions $i$ and $j$, and between the ON unit at position $i$ and the OFF unit at position $j$, respectively. $C_{\mathrm{ij}}^{\mathrm{D}}$ is positive when the activity of units of the same type covaries more strongly than that of units of different types and is negative when the opposite occurs. The average relative levels of correlation between units with receptive fields at different distances in the visual field were examined by means of the function $C^{\mathrm{D}}(\mathrm{d})=<C_{\mathrm{ij}}^{\mathrm{D}}$ $>_{|\mathrm{i}-\mathrm{j}|=\mathrm{d}}$, which evaluates the average correlation difference $C_{\mathrm{ij}}^{\mathrm{D}}$ among all pairs of cells at positions $i$ and $j$ at distance $d$ from each other. For simplicity, in the following we refer to $C^{\mathrm{D}}(d)$ as the correlation difference, implicitly assuming that a spatial averaging has taken place. The correlation difference is a useful tool for predicting the emerging patterns of connectivity in the presence of a Hebbian mechanism of synaptic plasticity (Miller (1994). The average separation at which $C^{\mathrm{D}}(d)$ changes sign is a key element in determining the spatial extent of the different subfields within the receptive fields of simple cells.

In some cases a first-order approximation of the correlation difference was evaluated by considering the LGN as a linear filter. This approximation neglects any effects of rectification. The correlation difference was evaluated by using the spatiotemporal correlation $r(d, t)$ of the input (the correlation function evaluated at time lag $t$ of the luminance of pixels located at a spatial lag $d$ ), or equivalently the input power spectrum $R(w, f)$, where $w$ and $f$ indicate spatial and temporal frequencies. Under the assumption of linearity, the correlation among geniculate cells of the same type $l(d, t)$ can be evaluated by the inverse Fourier transform of the power spectrum of LGN activity $l(d, t)=\mathscr{F}^{-1}\left(|H(w, f)|^{2} R(w, f)\right)$, where $H$ is the spatiotemporal Fourier transform of the LGN kernel [see, for example, Bendat and Piersol (1986)]. With the simplifying assumption that all cells are characterized by equal values of mean activity $\eta$ and standard deviation $\sigma$, the correlation difference was estimated as:

$$
C^{\mathrm{D}}(d)=\frac{2}{\sigma^{2}}\left(l(d, 0)-\eta^{2}\right)
$$

The results of the simulations were compared with the typical profile of a simple-cell receptive field as estimated by Jones and Palmer (1987b). According to the 2D Gabor filter model proposed by Jones and Palmer (1987a,b), the typical receptive field profile in the preferred direction $\theta$ is given by:

$$
w(r)=\left\langle\exp \left(r^{2} \frac{b^{2} \cos ^{2}(\theta)+a^{2} \sin ^{2}(\theta)}{2 a^{2} b^{2}}\right) \cos \left(-2 \pi F_{\mathrm{o}} r\right)\right\rangle,
$$

where $r$ indicates the distance from the center of the receptive field, and the averaging operation is over a number of cells with parameters shown in Table 1 of Jones and Palmer (1987b). The parameters $a$ and $b$ are the amplitudes of the Gaussian term along the two axes, $\theta$ gives the relative orientation of the Gaussian and wave modulations in the model, and $F_{\text {o }}$ is the wave frequency.

\section{RESULTS}

\section{LGN activity with static input presentation}

We first focus on the spatial characteristics of geniculate responses and consider the average activity of LGN cells in different situations. Figure 1 illustrates schematically some spatial factors that contribute to the emergence of correlated activity in the LGN and introduces the tools that we use to quantify such correlations. In this example we have measured the level of correlation between pairs of cells with receptive fields at different separations when a spot of light was presented as input. An important element in the resulting level of correlation is the polarity of the two cells (i.e., whether they are ON- or OFFcenter). As shown in Figure 1a, because geniculate cells tend to be coactive when the ON and OFF subregions of their receptive fields overlap, the correlation between pairs of cells of the same type decreases when the separation between their receptive fields is increased, whereas pairs of cells of opposite types tend to become more correlated. As a consequence, the correlation difference function, $C^{\mathrm{D}}(d)$, is positive at small separations and negative at large ones. Fig. $1 b$ illustrates an example of the dependence of the levels of correlation on the spatial structure of 


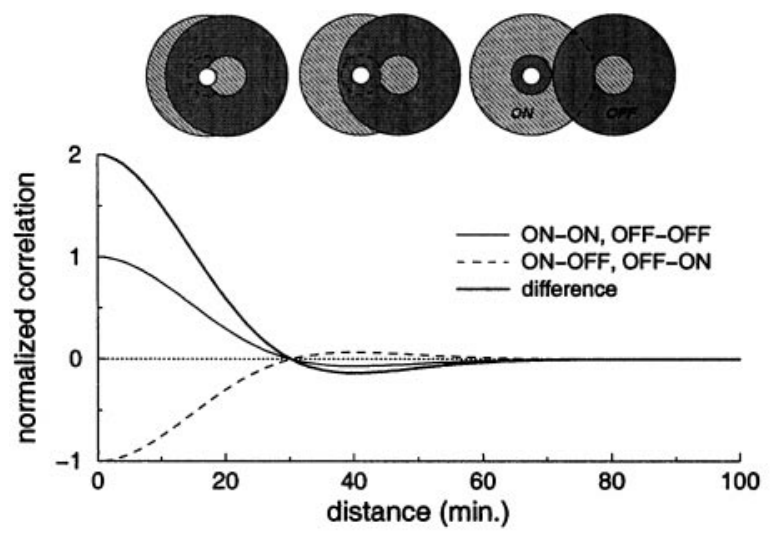

(a)

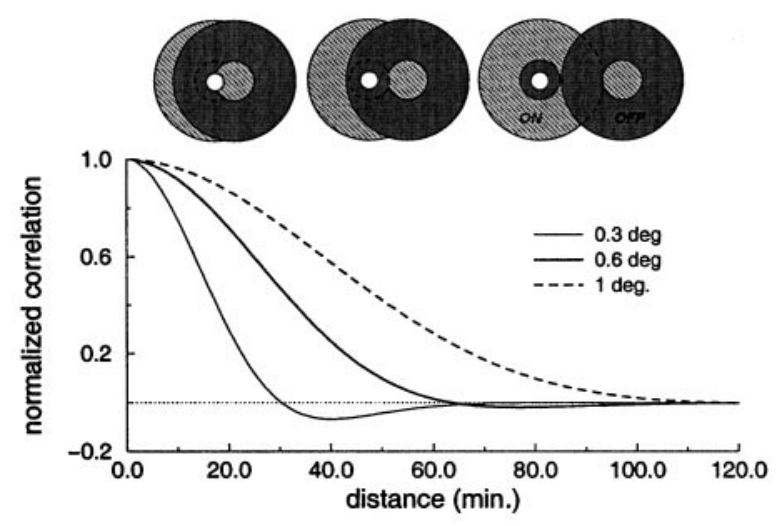

(b)

Figure 1. Analysis of some spatial factors affecting the correlation between the responses of two geniculate cells. Both graphs show the correlation coefficients or the correlation difference function, $C^{\mathrm{D}}(d)$, for pairs of cells with receptive fields at different separations. The icons on the top of each graph represent the positions of the receptive fields of the two cells at the corresponding separations along the $x$ axis. The bright dot marks the center of the spot of light. $a$, Effect of the polarity of the cells. The three curves represent the correlation coefficients for pairs of units of the same type $C^{\mathrm{s}}(d)$ (continuous thin line), units of opposite types $C^{\mathrm{o}}(d)$ (dashed line), and the correlation difference function $C^{\mathrm{D}}(d)=C^{\mathrm{s}}(d)-C^{\mathrm{o}}(d)($ bold line). $b$, Effect of the spatial structure of the visual input. Correlation difference functions measured in the presence of spots of light with three different sizes $(0.3,0.6$, and $1^{\circ}$ ). Positive (negative) values of $C^{\mathrm{D}}(d)$ indicate that the activity of LGN cells of the same (opposite) type covary more closely than the activity of cells of opposite (same) types.

the visual input. The correlation difference function changes significantly when the spot of light used for the stimulation is enlarged. In particular, $C^{\mathrm{D}}(d)$ becomes positive for $d$ in the range $30-70^{\prime}$ for a sufficiently large beam of light, indicating that when a large spot of light is present, pairs of cells with receptive fields at such separations possess stronger correlation if they have the same polarity, whereas the correlation between cells of opposite types predominates in the presence of a smaller spot.

In Figures 2 and 3, a similar analysis is presented for two richer types of visual input: retinal spontaneous activity and natural visual stimulation. Again, the data refer to the steady-state responses. Because little is known regarding the patterns of correlation in cat LGN during spontaneous activity, we have evaluated a first-order approximation on the basis of retinal inputs. Following the theory of linear filtering [see, for example, Bendat and Piersol (1986)], the autocovariance of LGN activity is given by the inverse Fourier transform of the output power spectrum $l_{\mathrm{e}}(x, y)=\mathscr{F}^{-1}\left(\left|H_{\mathrm{e}}\left(w_{\mathrm{x}}, w_{\mathrm{y}}\right)\right|^{2} R_{\mathrm{e}}\left(w_{\mathrm{x}}, w_{\mathrm{y}}\right)\right)$, where $R_{\mathrm{e}}\left(w_{\mathrm{x}}, w_{\mathrm{y}}\right)$ and $H_{\mathrm{e}}\left(w_{\mathrm{x}}, w_{\mathrm{y}}\right)$ are the Fourier transforms of the pattern of autocovariance of the inputs $r_{\mathrm{e}}(x, y)$ and the spatial geniculate kernel $h_{\mathrm{e}}(x, y)$ (see Materials and Methods). $l_{\mathrm{e}}(x, y)$ and $r_{\mathrm{e}}(x, y)$ are measures of the covariances among same-type geniculate and ganglion units with receptive fields located around eccentricity $e$ and spaced by $x$ and $y$ in the horizontal and vertical dimensions. As shown in Materials and Methods (Eq. 3), given $l_{\mathrm{e}}$ it is possible to estimate the correlation difference function. In Figure 2 we show the results for a typical LGN cell at $17^{\circ}$ of eccentricity $\left[\sigma_{\mathrm{c}}=18^{\prime}\right.$, $\sigma_{\mathrm{s}}=1.3^{\circ}$ (Linsenmeier et al., 1982)]. As a first approximation, the activity of retinal receptors was modeled as a white noise process; that is, the activation of each receptor was assumed to be independent from all the others. This approximation (data marked by filled squares in Fig. 2) is likely to underestimate the correlation difference of LGN cells in the cat, because it neglects any source of correlation other than the spatial organization of geniculate receptive fields. For example, positive correlations may already be present in the activity of neighboring retinal receptors, possibly originating from intrinsic connectivity (Vardi and Smith, 1996).

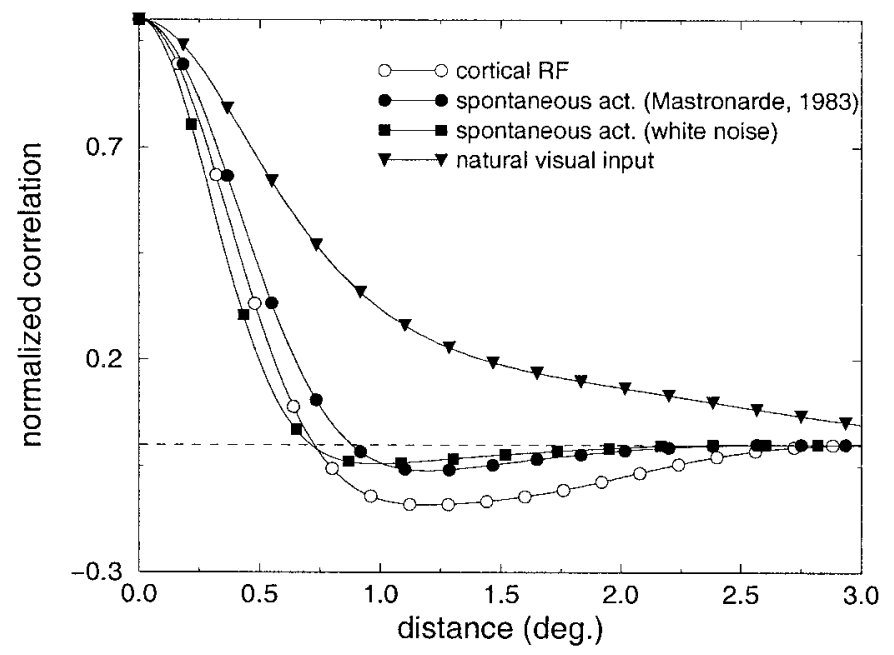

Figure 2. Predicted correlation difference functions of steady-state LGN activity evaluated around $17^{\circ}$ of visual eccentricity. $\mathbf{\square}$, Case of spontaneous activity (white noise approximation). $\mathbf{\bullet}$, Case of spontaneous activity (approximation with Mastronarde's data). $\mathbf{\nabla}$, Natural visual input. The curve marked by open circles is the average receptive field of a simple cell, as measured by Jones and Palmer (1987) shown here for comparison.

In a second approximation, $r_{\mathrm{e}}(x, y)$ was evaluated on the basis of Mastronarde's data on the correlated firing of ganglion cells in the cat retina (Mastronarde, 1983). Mastronarde's estimate of the correlation between the activity of two $\mathrm{X}$ cells at a relative distance $d$ [Mastronarde (1983), his Fig. 10] was approximated by the function $c_{\mathrm{e}}=15-3.75 s_{\mathrm{e}}(d)$, where $e$ is the visual eccentricity and $s_{\mathrm{e}}(d)$ indicates the distance expressed in spacings $\left[s_{\mathrm{e}}(d)=\right.$ $0.186 d \sqrt{N_{\mathrm{e}}} ; N_{\mathrm{e}}$ is the cell density at eccentricity $e$ as given by Hughes (1975)]. This second measurement (curve marked by filled circles in Fig. 2) is likely to provide an overestimate of the LGN autocovariance, because the spatial extent of positive correlations among the activity of ganglion cells is presumably larger than at the level of retinal receptors. For comparison, the average recep- 


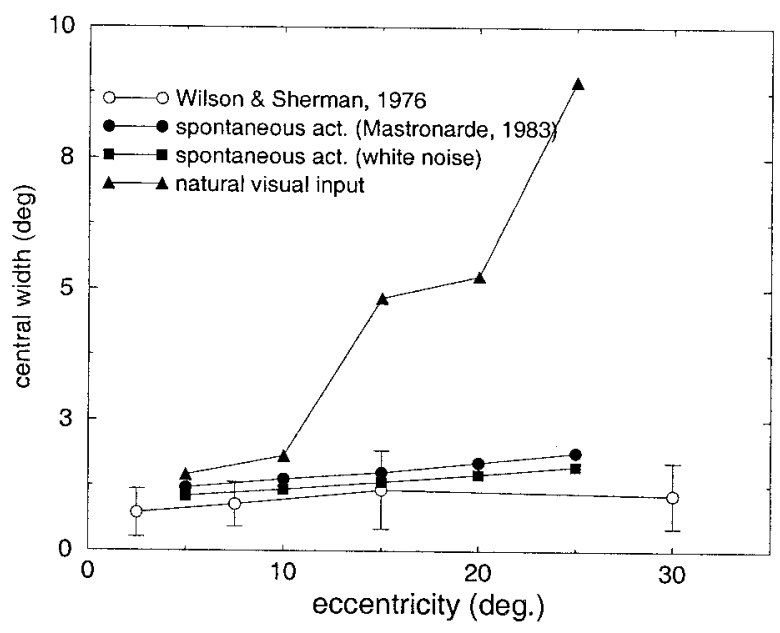

(a)

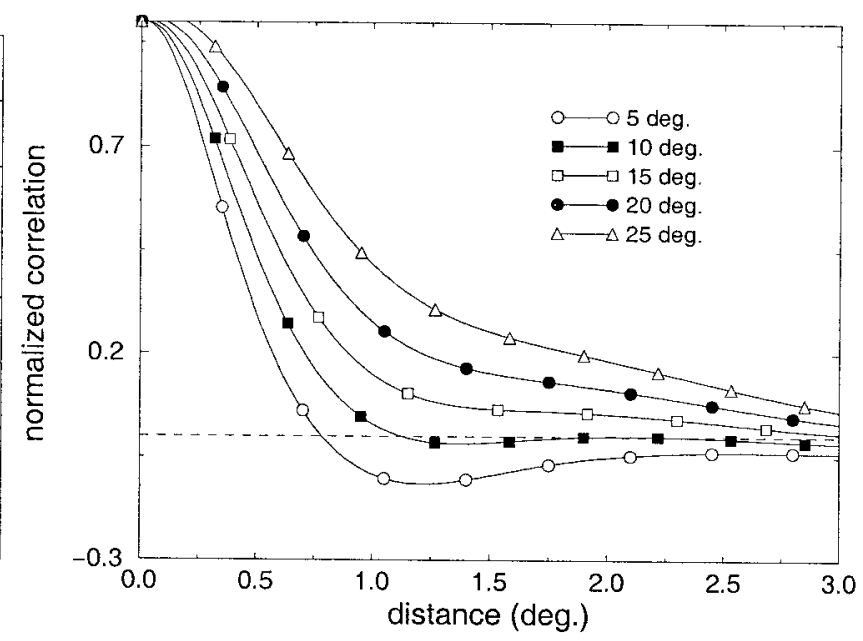

(b)

Figure 3. Analysis of the covariance patterns of steady-state LGN activity at different visual eccentricities. $a$, Comparison between the width of the larger subfield in the receptive field of simple cells at different eccentricities as measured by Wilson and Sherman (1976) (open circles) and the width of the central lobe of the correlation difference functions measured in the cases of spontaneous activity and natural visual input. Symbols as in Figure 2. $b$, Correlation difference functions measured in the presence of natural visual input at different eccentricities.

tive field profile of a simple cell within the central $5-25^{\circ}$ of the visual field is also shown. This prototypical cortical receptive field was taken from the measurements by Jones and Palmer (1987a,b). As illustrated by the graph, despite the fact that our method provides only a rough estimate of the correlation difference for the case of spontaneous activity, a close correspondence is present between these curves and the response profile of an average cortical simple cell, indicating that a Hebbian mechanism of synaptic plasticity can well account for the structure of simplecell receptive fields before eye opening.

What happens in the presence of natural visual input? A linear analysis similar to the case of spontaneous activity was performed using a database of 30 snapshot images of natural scenes. The mean power spectrum of our database was best approximated by $S(k)=A w^{-2.04}$, which is consistent with the results of several studies investigating the power spectrum of natural images (Field, 1994; Ruderman and Bialek, 1994; van der Schaaf and van Hateren, 1996). The mean correlation difference function measured when the input images were filtered by the spatial kernel of a LGN cell at $17^{\circ}$ of visual eccentricity is marked by filled triangles in Figure 2. This function was obtained from the mean autocovariance of LGN activity averaged along all possible orientations. Because of the wide spatial correlations of natural visual input, the estimated correlation difference did not change sign within the receptive field of a typical simple cell. That is, LGN cells of the same type were found to covary more closely than cells of opposite types at all separations within the receptive field of a simple cell. This result is not consistent with the putative role of a direct Hebbian/covariance model in the refinement of orientation selectivity after eye opening.

Figure 3 shows the results of the previous analysis for LGN cells at different visual eccentricities. The open circles in Figure $3 a$ represent the width of the largest subfield in the receptive field of cortical simple cells as measured by Wilson and Sherman (1976). The other curves represent the widths of the central lobe of the correlation difference functions (the spatial separation over which cells of the same type possess correlated activity, measured as the double of the point at which the correlation difference function intersects the zero axis) in the cases of spontaneous activity and natural visual input. As in Figure 2, a close correspondence was present between the experimental data and the subregion widths predicted by the correlation difference function in the case of spontaneous activity [in Fig. $3 a$, filled squares indicate white noise estimate, and filled circles indicate estimate on the basis of Mastronarde's data (Mastronarde, 1983)]. Conversely, a significant deviation between the two measurements was present in the case of natural visual input (Fig. $3 a$, filled triangles). The measured correlation differences at different visual eccentricities in the case of natural visual stimulation are shown in Figure $3 b$.

So far we have neglected the temporal evolution of neural responses. However, the activity of geniculate cells exhibits a large temporal variability, and several categories of geniculate cells have been identified on the basis of their dynamics. Depending on its own intrinsic dynamics, each LGN cell responds differently to changes in the visual input as a result of both the modifications of the surrounding environment and the motor behavior of the organism. In Figure 4 we analyze the levels of covariance in the activity of nonlagged $\mathrm{X}$ geniculate cells in the presence of natural time-varying input. Similar to the previous analysis, the correlation difference function was derived from the covariance of the activity of same-type cells, estimated by filtering the power spectrum of natural time-varying images with a typical spatiotemporal geniculate kernel. The results shown in Figure 4 refer to cells located around $17^{\circ}$ of visual eccentricity. The curve marked by filled circles in Figure 4 represents the correlation difference function evaluated when the power spectrum of time-varying natural images was approximated by considering visual scenes as collections of objects moving with a power-law distribution of velocities, as suggested by Dong and Atick (1995). An example in which the input power spectrum was experimentally evaluated on a sequence of images is also shown in Figure 4 (filled squares). In both cases, the positive values of 


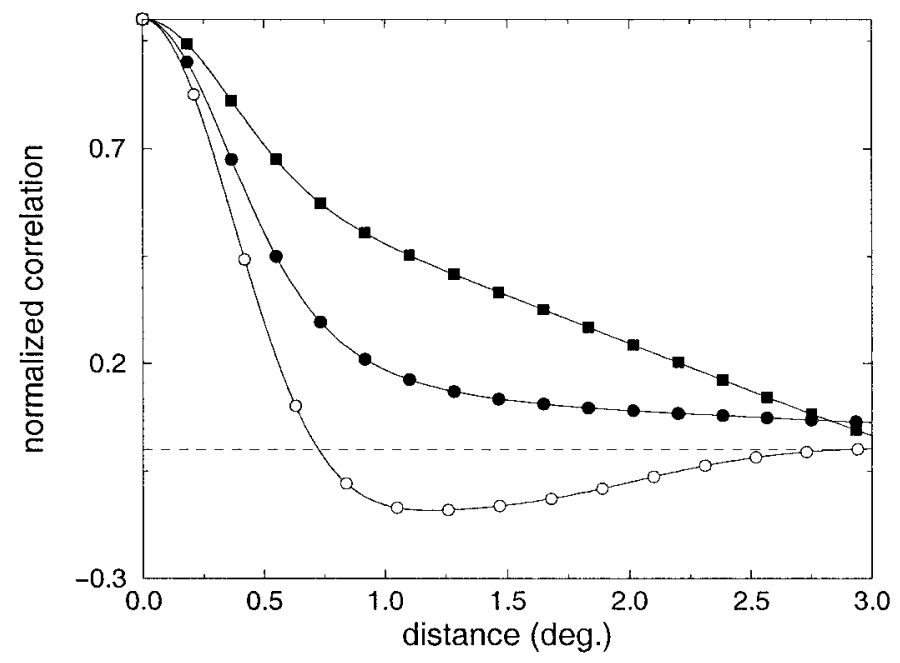

Figure 4. Correlation difference functions estimated in the presence of time-varying natural visual input. The curves with filled squares and circles are the results of two different approximations of the power spectrum of time-varying natural scenes (see Results for details). The curve marked by white circles is the average receptive field of a simple cell as measured by Jones and Palmer (1987) shown here for comparison.

the correlation difference functions indicate that cells of the same type tend to covary more strongly than cells of opposite polarity at all separations within the receptive field of a typical simple cell.

These results show that after eye opening the correlated activity of LGN cells derived from the static presentation of natural visual input does not match the structure of simple-cell receptive fields.

\section{LGN activity in the presence of eye movements}

In the remainder of this paper we focus on how changes in the visual input attributable to eye movements affect the correlated activity of geniculate cells. As described in Materials and Methods, both macroscopic (voluntary) and microscopic (involuntary) eye movements were modeled following the data available in the literature.

Figure 5 illustrates the results obtained when the images of the database were examined through random sequences of saccades. An example of the activation of two ON-center units with receptive fields separated by $0.8^{\circ}$ is shown in Figure $5 a$. Deviations of neural activity from their resting levels are shown in the top portion of the figure, and the $x$ and $y$ coordinates of the direction of gaze during the considered $5 \mathrm{sec}$ are plotted in the bottom part. The bar in the middle of the figure illustrates two different phases of the neural responses. The light portions of the bar correspond to fixation periods, defined as the time intervals starting $200 \mathrm{msec}$ after the acquisition of a fixation point and ending with the occurrence of a new saccade. Dark segments correspond to the transitory periods occurring between fixations. The measured correlation difference functions are shown in Figure $5 b$. The curves marked by filled symbols in Figure $5 b$ show the correlation differences obtained when the mean activity of LGN cells $\left(\bar{u}_{\mathrm{i}}\right.$ and $\bar{u}_{\mathrm{j}}$ in Eq. 2) were evaluated over different periods of observation $T$. Results presented later in the paper will illustrate the potential importance of this parameter. $C^{\mathrm{D}}$ did not change significantly when $T$ was reduced by two orders of magnitude (from $5 \mathrm{sec}$ to $100 \mathrm{msec}$ ), despite the fact that the averages of cell activity were estimated over several fixation points with $T=5 \mathrm{sec}$, and within a single fixation with $T=100 \mathrm{msec}$. Similar results were obtained when different phases of the neural responses were analyzed separately. The curves marked by open symbols in Figure $5 b$ are the correlation differences estimated with $T=100 \mathrm{msec}$ for the fixation (open triangles) and transitory phases (open circles). No significant differences were found in the case in which no modulation of cell activity was present at the time of saccades (gray symbols in Figure $5 b$ ). In all cases studied, the correlation difference function was positive at all of the separations that were considered.

A similar statistical analysis on the joint activity of pairs of geniculate cells for the case of microscopic eye movements is shown in Figure 6. In this experiment, 50 fixation points were randomly selected for each image. Each fixation lasted for a period of $5.5 \mathrm{sec}$, during which micromovements occurred and cell activity was evaluated. To isolate the contribution of micromovements with respect to that of saccades, correlation functions were evaluated starting $500 \mathrm{msec}$ after the onset of each fixation. Figure $6 a$ illustrates an example of the activity of two cells with receptive fields separated by $0.8^{\circ}$ during a sequence of micromovements. As before, the spatial parameters of the LGN kernel were chosen so as to model a cat geniculate cell at $17^{\circ}$ of visual eccentricity. The two curves marked by filled symbols in Figure $6 b$ illustrate the correlation difference functions obtained for the case of microsaccades ( filled circles), and for the joint presence of ocular drift and tremor ( filled triangles). Because in these experiments each fixation was maintained for a long period of time, the time window of observation, $T$, over which the averages of neural activity were evaluated was not an important parameter. For the curves in Figure $6 b, T=5 \mathrm{sec}$ was used. Interestingly, the correlation difference functions measured with the two types of micromovements were very similar. In both cases, strong correlations were found between the activity of LGN cells of opposite polarity with receptive fields located at separations larger than $0.6^{\circ}$, which is similar to the receptive field profile of a typical simple cell. Figure $6 c$ shows the correlation difference functions obtained at different visual eccentricities in the presence of tremor and ocular drift. The minimum separation between receptive fields necessary for observing strong levels of covariance between cells with opposite polarity increased with eccentricity, as illustrated by the points of zero-crossing of the different curves. Figure $6 d$ compares the widths of the central lobe of the estimated correlation functions at the different visual eccentricities with the size of the larger subfield in the receptive field of simple cells as measured by Wilson and Sherman (1976). In contrast to the results of Figure $3 a$, a close correspondence is now present between the spatiotemporal characteristics of LGN activity and the organization of simple-cell receptive fields.

Because the variation of retinal inputs is higher in the presence of saccades than with micromovements (in the simulations, the ratio between the SDs of the input signals in the two conditions was close to 2 ), it is to be expected that the correlations originating from macroscopic shifts of gaze will prevail when all movements are considered simultaneously. Figure 7 shows the results obtained when the images of the database were examined in the presence of both saccades and micromovements. Sequences of saccades were generated similarly to the experiments of Figure 5, and micromovements were overlapped during fixation periods. Figure $7 a$ shows an example of neural activity during a sequence of such movements. In this case, the time window over which the mean activation values are calculated becomes crucial, as illustrated in Figure $7 b$. In addition to considering the whole sequence of neural activity, geniculate responses in the fixation and transi- 


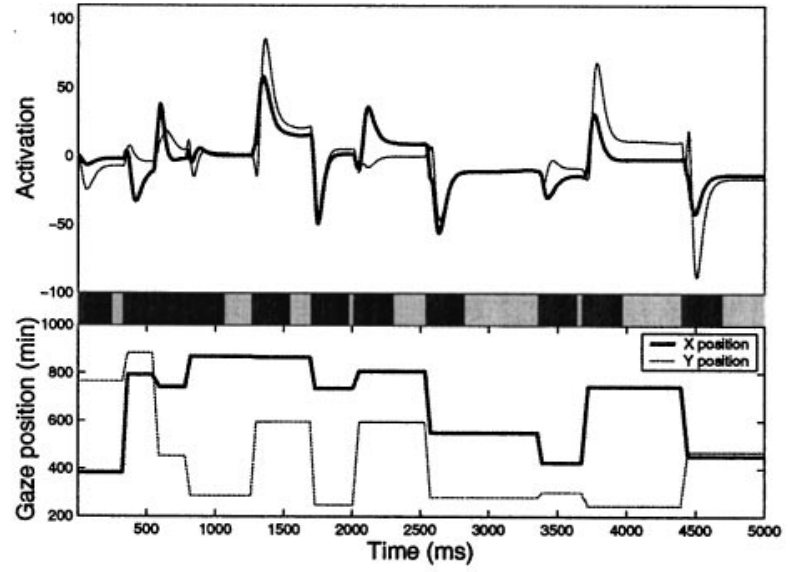

(a)

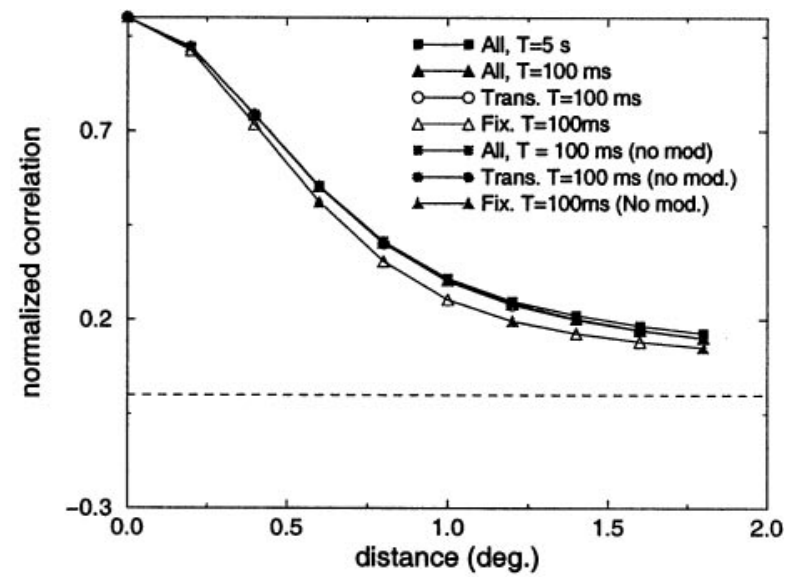

(b)

Figure 5. Analysis of the correlated activity of LGN cells in the case of saccades. $a$, Example of the response of two geniculate cells during a sequence of saccades. Neural activity is shown on the top. The curves on the bottom are the $x$ and $y$ coordinates of the direction of gaze. The bar in the middle indicates the two different phases of neural responses: fixation periods are marked by the light segments, transitory periods by dark segments. The receptive fields of the two cells were $0.8^{\circ}$ apart. $b$, Correlation difference functions obtained in the presence of saccades. The results obtained over different time windows and for different phases of the responses are shown in the presence and absence of saccadic modulation of neural activity.

tory phases were also analyzed separately. When the whole response was considered without differentiating between the two phases, the correlation difference was positive at all of the separations considered, independent of the length of the time window of observation. A similar result was also found for the transitory phase. Conversely, strong correlations between the activity of LGN cells of opposite polarity were found during fixation periods, when the covariances were evaluated over a time window of $100 \mathrm{msec}$. No significant differences were found when neural activity was not modulated during saccades. These results indicate that in the presence of eye movements, a Hebbian scheme of plasticity can account for the organization of simple-cell receptive fields, if synaptic changes are prevented during saccades and the induction of plasticity is sensitive to the covariance of neural activity occurring within a time window smaller than the average fixation.

What factors are responsible for the differences in $C^{\mathrm{D}}$ measured in the presence of saccades and micromovements? Because the kernels used to model LGN cells were identical in the two conditions, corresponding statistical differences should be present in the input visual patterns. An obvious difference between saccades and microscopic eye movements is the spatial scale of the visual analysis involved. When saccades of random amplitude covering the whole input image are performed, retinal inputs depend on the global statistical properties of the visual scene. The amplitude of saccades is on average significantly larger than the separations among receptive fields considered in this paper. Because the broad correlations within natural visual scenes, when areas with different brightness in the input image are swept across receptive fields, visual inputs to nearby retinal receptors tend to vary in a similar way. Given the high speed of saccades, the mean value of such inputs is close to the average brightness of the image even when evaluated over relatively short time windows. As a result, the covariance of the inputs to retinal receptors at different separations evaluated on a time window $T$ is largely determined by the correlation structure of static natural images for a wide range of $T$. The situation is significantly different in the presence of micromovements, both microsaccades and the combination of ocular drift and tremor. In this case, if a time window shorter than the average fixation is chosen, the broad correlations of luminance typical of natural visual images tend to be discounted by the operation of covariance, because they are largely reflected by the mean levels of cell activity over the considered window. In the presence of a short window of observation, the remaining temporal covariances of input activity depend on the local statistical characteristics of the images around each point, i.e., the covariances in pixel intensities at a spatial scale determined by the extent of micromovements. Because broad luminance covariances are subtracted out by using short time windows, the correlation difference function of geniculate activity tends to be determined by the spatial structure of geniculate cell receptive fields.

In Figure $8 a$ the correlation coefficients evaluated over 100 msec between inputs to pairs of retinal receptors at different separations are plotted for the two situations of all movements (filled circles) and saccades only (open circles). These data were calculated on a set of 6000 observations, each composed of a time period of $5.5 \mathrm{sec}$ in which the different types of eye movements occurred. The simulated trains of retinal inputs were divided into chunks of $100 \mathrm{msec}$ over which covariances were evaluated. In the presence of ocular drift and tremor, retinal inputs were independent for separations $>0.25^{\circ}$, whereas during saccades, retinal inputs remained correlated over large separations. As a result of the reduction in the extent of spatial correlations, in the presence of micromovements the correlation difference evaluated over a similar time window was largely determined by the characteristics of the geniculate kernel. This is shown in Figure $8 b$, where the correlation difference functions measured at two different visual eccentricities are compared with the autocovariances of the geniculate filters in the two positions. Figure $8 a$ also shows the patterns of covariance measured using running averages (filled and open triangles for the two experimental conditions) as in the experiments in Figure 7. These curves are almost identical to those derived on the basis of the covariance on the same time interval. It is important to notice that the data in Figure $8 a$ do not 


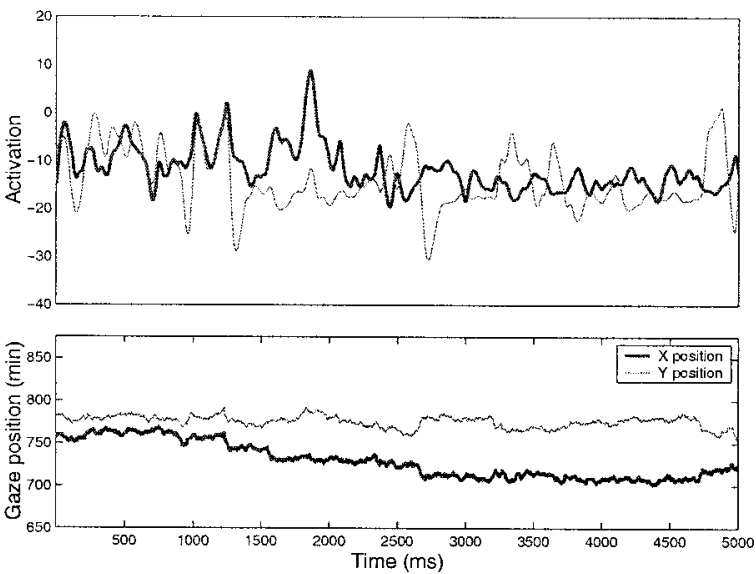

(a)

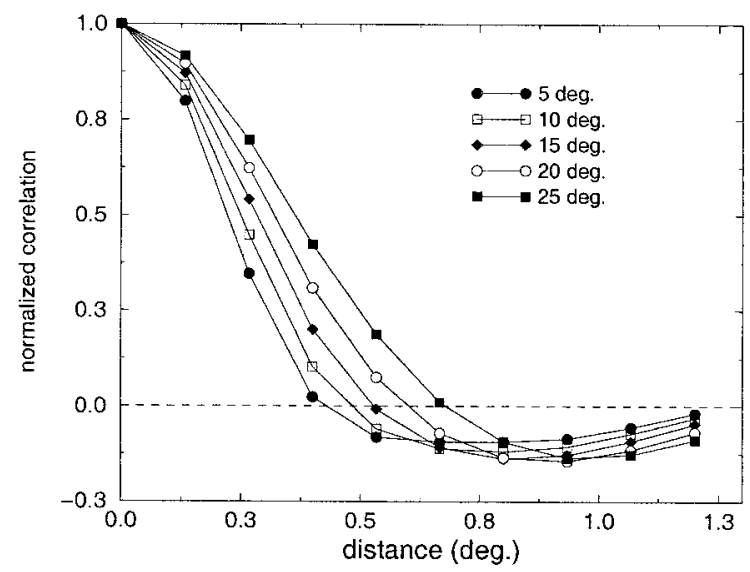

(c)

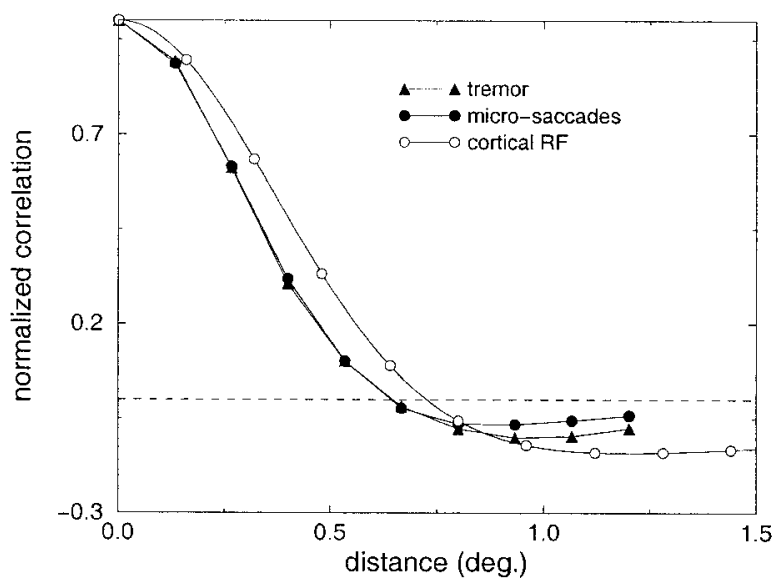

(b)

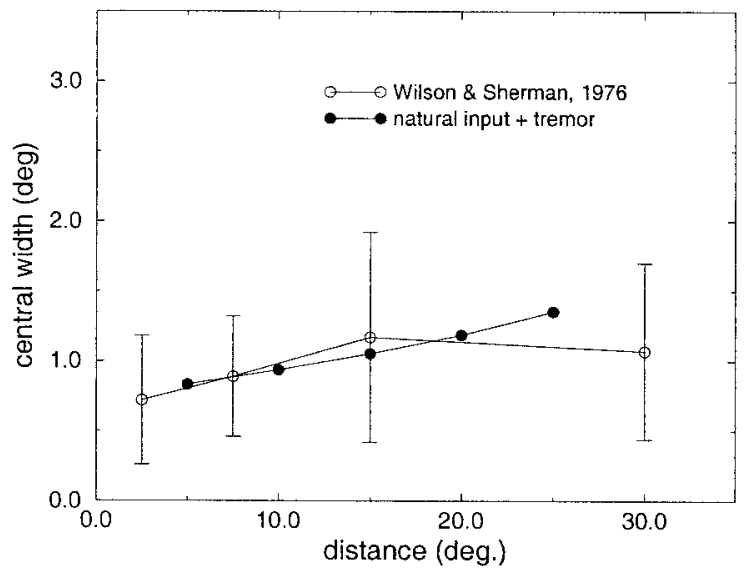

(d)

Figure 6. Analysis of the correlated activity of LGN units in the presence of different types of microscopic eye movements. $a$, Example of the activation of two cells (top graph) during a sequence of microsaccades and tremor (bottom graph). $b$, Correlation differences in the case of microsaccades ( $)$ and ocular drift and tremor $(\boldsymbol{\Delta}) . c$, Correlation differences evaluated at different visual eccentricities. $d$, Comparison between the width of the central lobe of the correlation difference function and the width of the larger subfield in the receptive fields of simple cells at different eccentricities (Wilson and Sherman, 1976).

depend on the duration of the experiment, but they are exclusively a function of the window $T$ on which covariances are evaluated or running averages are performed.

In the simulations described so far we have made the assumption that variations in geniculate activity occurred within the linear range. However, in reality LGN responses often deviate from these linear approximations. The main source of nonlinearity is known to originate from the rectification of activity, an operation that eliminates negative firing rates ([. $]_{\theta}$ in Eq. 1). Because neural responses can be significantly different after rectification, it is important to study the patterns of covarying activity in the presence of such operation. Figure $9 a$ shows the effects of different levels of rectification when saccades (open symbols) and tremor ( filled symbols) were selectively present. No significant changes were measured in the correlation difference even in the case of a $100 \%$ rectification, when the range of negative responses was completely eliminated. Figure $9 b$ shows the corre- lation difference functions obtained for a $100 \%$ rectification when both saccades and micromovements were simultaneously present. As shown by the graphs, the patterns of covariance were similar to those of Figure $7 b$ obtained in the absence of rectification.

\section{DISCUSSION}

In this paper we have used computer modeling to study the correlated activity of LGN cells when images of natural scenes were scanned to replicate cat eye movements. In our simulations, the measured patterns of covarying activity matched simple-cell receptive fields as required by a direct Hebbian/covariance mechanism of synaptic plasticity in the presence of micromovements, but not when natural scenes were statically presented or scanned through sequences of saccades. These results suggest a developmental role for the microscopic eye movements that occur during visual fixations. 


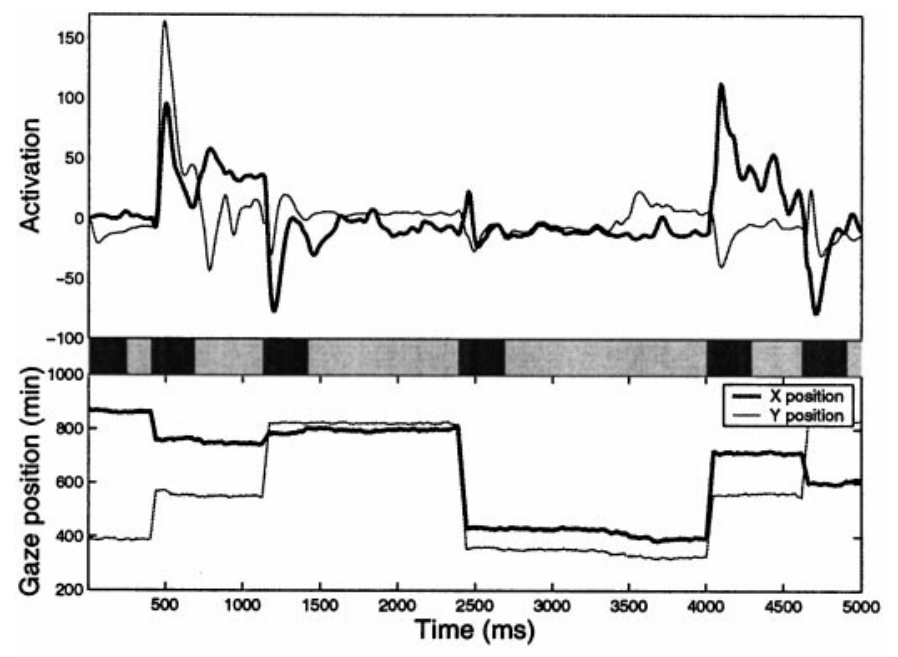

(a)

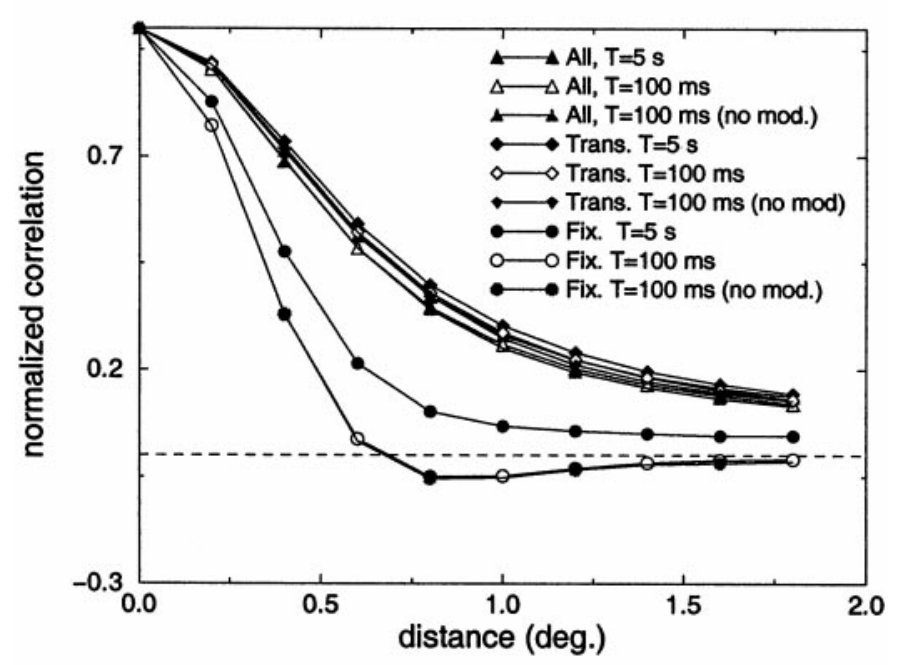

(b)

Figure 7. Analysis of the correlated activity of LGN units when both macroscopic and microscopic eye movements are simultaneously present. $a$, Example of the response of two geniculate units with receptive fields $0.8^{\circ}$ apart (top) during a sequence of eye movements including macrosaccades and micromovements (bottom). The bar in the middle indicates the two phases in which responses have been subdivided: fixation periods are marked by the light segments, transitory periods by dark segments. $b$, Correlation difference functions obtained when the different phases of the response were analyzed separately. Data obtained in the absence of modulation of activity during saccades are also shown.

\section{Eye movements and the development of cortical orientation selectivity}

Although the role of visual experience in the development of orientation selectivity has been extensively investigated, relatively few studies have focused on whether eye movements contribute to the development of the responses of cortical cells. Our finding that the patterns of LGN activity with static presentation of natural images did not match the spatial structure of the receptive fields of simple cells is in agreement with the hypothesis that exposure to pattern vision per se is not sufficient to account for a normal visual development (Buisseret et al., 1978; Freeman and Bonds, 1979; Gary-Bobo et al., 1986; Buisseret, 1995).

A main assumption of this study is that the refinement and maintenance of orientation selectivity after eye opening is mediated by a Hebbian/covariance process of synaptic plasticity (Stent, 1973; Changeux and Danchin, 1976). The term Hebbian is used here with a generalized meaning to indicate the family of algorithms in which modifications of synaptic efficacies occur on the basis of the patterns of input covariances (Sejnowski, 1977; Montague et al., 1991; Cruikshank and Weinberger, 1996). Although no previous theoretical study has investigated the influence of eye movements on the development of orientation selectivity, some models have shown that schemes of synaptic modifications based on the correlated activity of thalamic afferents can account well for the segregation of ON- and OFF-center inputs before eye opening in the presence of suitable patterns of spontaneous activity (Linsker, 1986; Miyashita and Tanaka, 1992; Miller, 1994). By showing that, during fixation, the spatiotemporal structure of visually driven geniculate activity is compatible with the structure of simple-cell receptive fields, the results of the present study extend the plausibility of such schemes to the period after eye opening in which exposure to pattern vision occurs.

The results of our simulations appear to contrast with those of some modeling studies showing that Hebbian mechanisms of plasticity lead to the development of oriented receptive fields when natural images are statically presented (Barrow et al., 1996; Shouval et al., 1997). Although these simulations produced receptive fields that qualitatively resembled those of cortical simple cells, a quantitative comparison like the one in this paper was not performed. It should also be noted that computational studies have shown that the segregation and/or clustering of synaptic inputs may depend on factors other than the patterns of input correlations (Goodhill, 1993; Miller and MacKay, 1994). In particular, a segregation of afferents could occur even in the presence of predominant correlations among cells of the same type, if a rule of synaptic modification based on subtractive normalization is adopted (Miller and MacKay, 1994). However, subtractive normalization has been questioned on the grounds of biological plausibility (Elliott and Shadbolt, 1998). Moreover, our finding of strong covarying activity among cells of opposite polarity during fixation eliminates the need to postulate a specific type of normalization.

As in any modeling study, our results depend on the degree of accuracy with which biological signals are replicated. A number of factors were not present in the model, including sources of correlated activity in the LGN other than shared visual stimulation (Sillito et al., 1994; Alonso et al., 1996; Neuenschwander and Singer, 1996; Dan et al., 1998), and changes in the spatiotemporal characteristics of geniculate responses during growth (Braastad and Heggelund, 1985; Cai et al., 1997). Considerations of this sort emphasize the need to validate the predictions of our study with in vivo experiments. In any case, the results presented in this paper were robust with respect to a number of factors. When the spatial characteristics of simulated geniculate units were changed to replicate the properties of cells in different regions of the visual field, results consistent with physiological data were obtained. Measured patterns of correlation were found to be tolerant with respect to the fine characteristics of simulated eye movements, modulation of activity, and degree of rectification. 


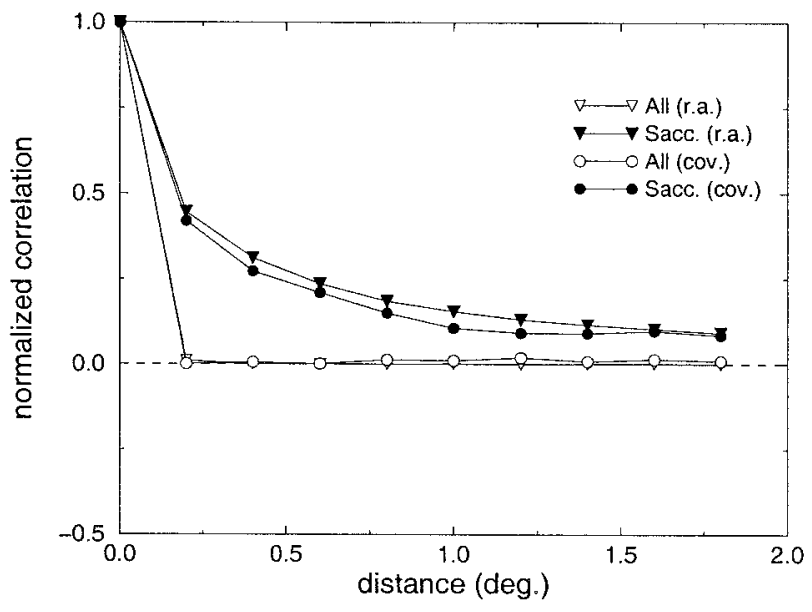

(a)

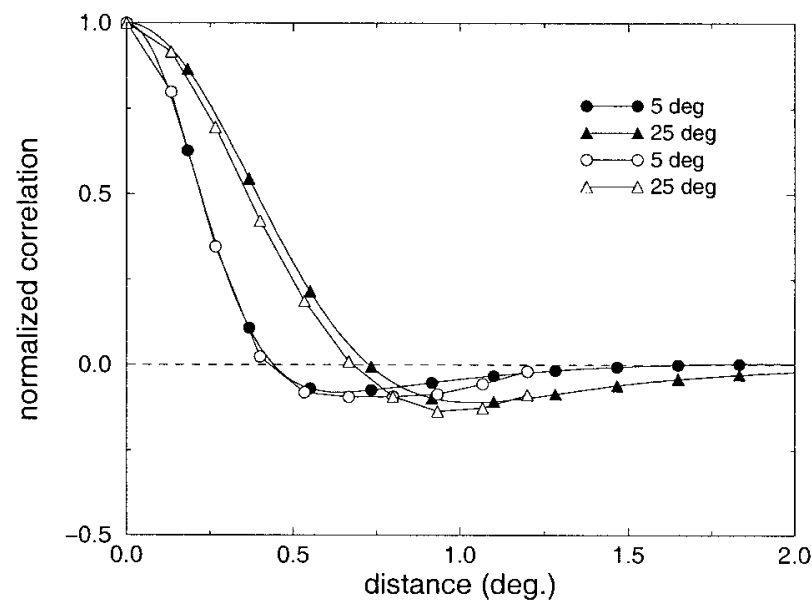

(b)

Figure 8. a, Second-order statistics of the visual input measured in the presence of all movements (open symbols) or saccades only ( filled symbols). See Results for details. $b$, Comparison between the autocovariances of typical LGN receptive fields ( filled symbols) and the correlation difference functions (open symbols) obtained at 5 and $25^{\circ}$ of visual eccentricity in the presence of micromovements.

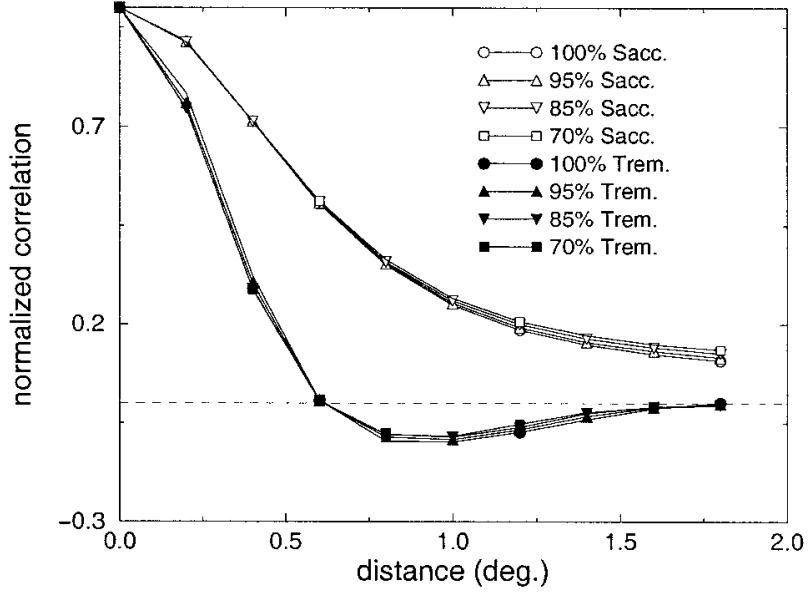

(a)

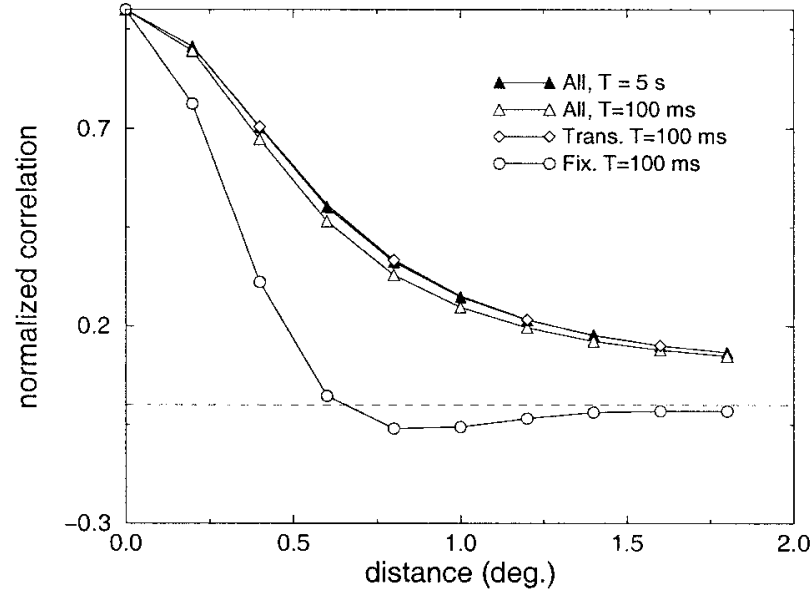

(b)

Figure 9. Analysis of the correlated activity of LGN units in the presence of different levels of rectification. $a$, Correlation difference functions obtained in the cases of saccades and tremor. $b$, Correlation difference functions obtained with a $100 \%$ rectification when both saccades and micromovements were present.

\section{Predictions}

A first prediction of this study is that the experience of pattern vision in the absence of microscopic eye movements may lead to an impairment in the development of orientation selectivity. Microscopic eye movements occurring during fixation have been studied mainly in humans (Ditchburn, 1973; Steinman et al., 1973), but they are known to occur also in monkey (Skavenski et al., 1975; Leopold and Logothetis, 1998) and cat (Conway et al., 1981; Gomez et al., 1986). Although experiments aimed at selectively analyzing the developmental role of different types of eye movements have not been performed, it is interesting to note that a reduction in the percentage of orientation-selective cells and an increase in the size of simple-cell receptive fields have been found in cats reared under low-frequency stroboscopic illumination
(Cynader et al., 1973; Olson and Pettigrew, 1974; Cremieux et al., 1987), a condition that alters the sampling of the visual environment similar to that occurring during an oculomotor manipulation. It has been suggested that such impairment in the development of orientation selectivity could be caused by the prolonged intervals of darkness that make this condition similar to dark rearing. The results described in this paper suggest the possible alternative interpretation that low-frequency stroboscopic illumination could operate by eliminating the effect of micromovements and constraining the spatiotemporal structure of the visual input to be similar to that recorded during sequences of saccades. In the oculomotor behavior of strobe-reared kittens, profound changes selectively affecting fixation eye movements have been reported (Conway et al., 1981). 
Additional predictions of this study concern the developmental mechanisms of plasticity. A first observation is that synaptic changes should be affected by the covarying activity of presynaptic afferents evaluated over time windows smaller than the average fixation period. Indeed, suitable patterns of covariance were not present in the model when evaluated over longer windows. Although little is known about the length of such intervals, they should not be confused with the longer period of time over which previous postsynaptic activity may influence synaptic plasticity (Huang et al., 1992; Kirkwood et al., 1996). A second observation is that a gating of synaptic plasticity should be present during macroscopic redirection of gaze to prevent a degradation in the segregated structure of geniculate afferents. It has been proposed that experience-dependent modifications of simple-cell receptive fields may be gated by proprioceptive inputs (Buisseret and Singer, 1983). This proposal is consistent with the impairment found in the reestablishment of orientation selectivity in darkreared kittens exposed to a few hours of visual experience when proprioceptive inputs from extraocular eye muscles were eliminated (Buisseret and Gary-Bobo, 1979; Trotter et al., 1981). There are several plausible ways in which such gating of plasticity could be accomplished. One possibility is that synaptic modifications are minimized during saccades after a reduction of the correlated activity of presynaptic and postsynaptic elements. Given the multiplication of presynaptic and postsynaptic activity postulated by the Hebbian/covariance rules, even a $10 \%$ reduction in geniculate activity (Lee and Malpeli, 1998) could be sufficient to preserve segregation if a depression of cortical activity is simultaneously present. A transient depression of neural activity in the striate cortex in conjunction with saccades has been reported (Duffy and Burchfiel, 1975; Toyama et al., 1984). Alternatively, a gating of synaptic plasticity could be achieved by affecting the chain of cellular events after a change in the level of correlation, independent of any direct effect on the correlation itself.

A stimulating hypothesis is that proprioceptive gating of plasticity is mediated by the activity of diff use-projecting neuromodulatory systems, such as the monoaminergic and cholinergic systems (Singer, 1982). A large body of evidence indicates that the neuromodulators released by these systems play a role in longterm plasticity (Kasamatsu et al., 1979; McGaugh, 1989; Artola and Singer, 1993; Bear and Kirkwood, 1993; Gu and Singer, 1993, 1995), and it is also well established that the activity of these systems is higher in the presence of salient sensory events and in states of arousal (Foote and Morrison, 1987; Aston-Jones et al., 1991; Marrocco et al., 1994; Robbins and Everitt, 1995), conditions likely to occur in conjunction with saccades. In this view, the activation of diffuse-projecting modulatory systems in correspondence with a shift of gaze (Steinfels et al., 1983; Aston-Jones et al., 1991) would enable the occurrence of long-term synaptic changes during the fixation periods after each saccade. We have recently proposed a similar scheme of plasticity, in which synaptic changes are gated by the activity of diffuse-projecting neuromodulatory systems so as to occur after a redirection of gaze, to account for the registration of multimodal maps of space in the optic tectum of the barn owl (Rucci et al., 1997).

\section{Extensions and future directions}

The simulations described in this paper were designed to replicate the changes in the visual input that occur during eye movements in the cat. It remains to be studied whether the visual changes induced by other behaviors (for example, head move- ments and navigation) could have a similar developmental role. It should also be noted that smooth pursuit eye movements were not considered in this study. Smooth pursuit has been observed in naive cats only in complete darkness and for low target velocities $\left(\sim 2^{\circ} / \mathrm{sec}\right)$ (Missal et al., 1995). In the light, cats appear to pursue moving targets with saccades triggered every time the target moves too far from the area centralis. It is conceivable that smooth pursuit may play an important role in the visual development of different species possessing such eye movements.

Although in this paper we have focused on the development of thalamocortical projections, oculomotor activity could also directly influence the development of intracortical connectivity. During fixation, micromovements could foster the development of long-range patchy connectivity by correlating the activity of cells with similar response characteristics. Hebbian mechanisms of plasticity have been proposed to be responsible for the development of the patchy organization of horizontal cortical connections (Katz and Callaway, 1992; Löwel and Singer, 1992; Ruthazer and Stryker, 1996; Troyer et al., 1998). In addition, in monkey striate cortex both depression and facilitation of neural responses have been observed after microsaccades (Leopold and Logothetis, 1998).

Ocular movements are a common feature of the visual system of different species. It should not come as a surprise that a trace of their existence can be found even in some of the most basic properties of neurons in the early stages of the visual system, such as orientation selectivity. Further studies are needed to investigate whether similar traces can be found in other features of visual neural responses.

\section{REFERENCES}

Albus K, Wolf K (1984) Early postnatal development of neuronal function in the kitten's visual cortex: a laminar analysis. J Physiol (Lond) 348:153-185.

Alonso JM, Usrey WM, Reid RC (1996) Precisely correlated firing in cells of the lateral geniculate nucleus. Nature 383:815-819.

Artola A, Singer W (1993) Long-term depression of excitatory synaptic transmission and its relationship to long-term potentiation. Trends Neurosci 16:480-487.

Aston-Jones G, Chiang C, Alexinsky T (1991) Discharge of noradrenergic locus coeruleus neurons in behaving rats and monkeys suggests a role in vigilance. In: Neurobiology of the locus coeruleus (Barnes CD, Pompeiano O, eds), pp 501-519), Amsterdam: Elsevier.

Barrow HG, Bray AJ, Budd JML (1996) A self-organizing model of "color blob" formation. Neural Comput 8:1427-1448.

Bear MK, Kirkwood A (1993) Neocortical long-term potentiation. Curr Opin Neurobiol 3:197-202.

Bendat JS, Piersol AG (1986) Random data: analysis and measurement procedures. New York: Wiley.

Blakemore C, Van Sluyters RC (1975) Innate and environmental factors in the development of the kitten's visual cortex. J Physiol (Lond) 248:663-716.

Braastad BO, Heggelund P (1985) Development of spatial receptive field organization and orientation selectivity in kitten striate cortex. J Neurophysiol 53:1158-1178.

Buisseret P (1995) Influence of extraocular muscle proprioception on vision. Physiol Rev 75:323-338.

Buisseret P, Gary-Bobo E (1979) Development of visual cortical orientation specificity after dark-rearing: role of extraocular proprioception. Neurosci Lett 13:259-263.

Buisseret P, Imbert M (1976) Visual cortical cells: their developmental properties in normal and dark-reared kittens. J Physiol (Lond) 255:511-525.

Buisseret P, Singer W (1983) Proprioceptive signals from extraocular muscles gate experience-dependent modifications of receptive fields in the kitten visual cortex. Exp Brain Res 51:443-450.

Buisseret P, Gary-Bobo E, Imbert M (1978) Ocular motility and recovery of orientational properties of visual cortical neurons in dark-reared kittens. Nature 272:816-817. 
Cai D, DeAngelis GC, Freeman RD (1997) Spatiotemporal receptive field organization in the lateral geniculate nucleus of cats and kitten. J Neurophysiol 78:1045-1061.

Changeux JP, Danchin A (1976) Selective stabilization of developing synapses as a mechanism for the specification of neuronal networks. Nature 264:705-712.

Chapman B, Stryker MP (1993) Development of orientation selectivity in ferret visual cortex and effects of deprivation. J Neurosci 13:5251-5262.

Chapman B, Zahs KR, Stryker MP (1991) Relation of cortical cell orientation selectivity to alignment of receptive fields of the geniculocortical afferents that arborize within a single orientation column in ferret visual cortex. J Neurosci 11:1347-1358.

Chapman B, Stryker MP, Bonhoffer T (1996) Development of orientation preference maps in ferret primary visual cortex. J Neurosci 16:6443-6453.

Chung S, Ferster D (1998) Strength and orientation tuning of the thalamic input to simple cells revealed by electrically evoked cortical suppression. Neuron 20:1177-1189.

Conway JL, Timberlake GT, Skavenski AA (1981) Oculomotor changes in cats reared without experiencing continuous retinal image motion. Exp Brain Res 43:229-232.

Crair MC, Gillespie DC, Stryker MP (1998) The role of visual experience in the development of columns in cat visual cortex. Science 279:566-570.

Cremieux J, Orban GA, Duysens J, Amblard B (1987) Response properties of area 17 neurons in cats reared in stroboscopic illumination. J Neurophysiol 57:1511-1535.

Cruikshank SJ, Weinberger NM (1996) Evidence for the Hebbian hypothesis in experience-dependent physiological plasticity of neocortex: a critical review. Brain Res Rev 22:191-228.

Cynader M, Berman N, Hein A (1973) Cats reared in stroboscopic illumination: effects on receptive fields in visual cortex. Proc Natl Acad Sci USA 70:1353-1354.

Dan Y, Alonso J, Usrey W, Reid R (1998) Coding of visual information by precisely correlated spikes in the lateral geniculate nucleus. Nat Neurosci 6:501-507.

Ditchburn RW (1973) Eye-movements and visual perception. Oxford: Clarendon.

Dong DW, Atick JJ (1995) Statistics of natural time-varying images. Network Comput Neural Sys 6:345-358.

Duffy FH, Burchfiel JL (1975) Eye movement-related inhibition of primate visual neurons. Brain Res 89:12-132.

Eizenman M, Hallett P, Frecker RC (1985) Power spectra for ocular drift and tremor. Vision Res 25:1635-1640.

Elliott T, Shadbolt NR (1998) Competition for neurotrophic factors: ocular dominance columns. J Neurosci 18:5850-5858.

Ferster D, Chung S, Wheat H (1996) Orientation selectivity of thalamic input to simple cells of cat visual cortex. Nature 380:249-252.

Field DJ (1994) What is the goal of sensory coding. Neural Comput 6:559-601.

Foote SL, Morrison JH (1987) Extrathalamic modulation of cortical function. Annu Rev Neurosci 10:67-95.

Freeman RD, Bonds AB (1979) Cortical plasticity in monocularly deprived immobilized kittens depends on eye movement. Science 206:1093-1095.

Fregnac Y, Imbert M (1978) Early development of visual cortical cells in normal and dark-reared kittens: relationship between orientation selectivity and ocular dominance. J Physiol (Lond) 278:27-44.

Fregnac Y, Shulz D, Thorpe S, Bienenstock E (1992) Cellular analogs of visual cortical epigenesis. I. Plasticity of orientation selectivity. J Neurosci 12:1280-1300.

Gary-Bobo E, Milleret C, Buisseret P (1986) Role of eye movements in developmental process of orientation selectivity in the kitten visual cortex. Vision Res 26:557-567.

Gomez C, Canals J, Torres B, Delgado-Garcia JM (1986) Analysis of the fluctuations in the interspike intervals of abducens nucleus neurons during ocular fixation in the alert cat. Brain Res 381:401-404.

Goodhill GJ (1993) Topography and ocular dominance: a model exploring positive correlations. Biol Cybern 69:109-118.

Gu Q, Singer W (1993) Effects of intracortical inf usion of anticholinergic drugs on neuronal plasticity in kitten visual cortex. Eur J Neurosci 5:475-485.

Gu Q, Singer W (1995) Involvement of serotonin in developmental plasticity of kitten visual cortex. Eur J Neurosci 7:1146-1153.
Harris CM, Hainline L, Abramov I, Lemerise E, Camenzuli C (1988) The distribution of fixation durations in infants and naive adults. Vision Res 28:419-432.

Hein A, Vital-Durand F, Salinger W, Diamond R (1979) Eye movements initiate visual-motor development in the cat. Science 204:1321-1322.

Hirsch HVB (1985) The role of visual experience in the development of cat striate cortex. Cell Mol Neurobiol 5:103-121.

Huang YY, Colino A, Selig DK, Malenka RC (1992) The influence of prior synaptic activity on the induction of long-term potentiation. Science 255:730-733.

Hubel DH, Wiesel TN (1962) Receptive fields, binocular interaction and functional architecture in the cat's visual cortex. J Physiol (Lond) 160:106-154

Hughes A (1975) A quantitative analysis of the cat retinal ganglion cell topography. J Comp Neurol 163:107-128.

Jones JP, Palmer LA (1987a) An evaluation of the two-dimensional gabor filter model of simple receptive fields in cat striate cortex. J Neurophysiol 58:1233-1257.

Jones JP, Palmer LA (1987b) The two-dimensional spatial structure of simple receptive fields in cat striate cortex. J Neurophysiol 58:1187-1211.

Kasamatsu T, Pettigrew JD, Ary ML (1979) Restoration of visual cortical plasticity by microperfusion of norepinephrine. J Comp Neurol 185:163-182.

Katz LC, Callaway EM (1992) Development of local circuits in mammalian visual cortex. Annu Rev Neurosci 15:31-56.

Kirkwood A, Rioult MG, Bear MF (1996) Experience-dependent modification of synaptic plasticity in the visual cortex. Nature 381:526-528.

Kuboki T (1957) Studies on discharge intervals of single motor units in human extraocular muscles. Tokohu J Exp Med 66:91-95.

Lee D, Malpeli JG (1998) Effect of saccades on the activity of neurons in the cat lateral geniculate nucleus. J Neurophysiol 79:922-936.

Leopold DA, Logothetis NK (1998) Microsaccades differentially modulate neural activity in the striate and extrastriate visual cortex. Exp Brain Res 123:341-345.

Linsenmeier RA, Frishman LJ, Jakiela HG, Enroth-Cugell C (1982) Receptive field properties of $\mathrm{x}$ and $\mathrm{y}$ cells in the cat retina derived from contrast sensitivity measurements. Vision Res 22:1173-1183.

Linsker R (1986) From basic network principles to neural architecture: emergence of orientation-selective cells. Proc Natl Acad Sci USA 83:8390-8394.

Löwel S, Singer W (1992) Selection of intrinsic horizontal connections in the visual cortex by correlated neuronal activity. Science 255:209-212.

Marrocco RT, Witte EA, Davidson MC (1994) Arousal systems. Curr Opin Neurobiol 4:166-170.

Mastronarde DN (1983) Correlated firing of cat retinal ganglion cells. I. Spontaneously active inputs to $\mathrm{X}$ and $\mathrm{Y}$ cells. J Neurophysiol 49:303-323.

McGaugh JL (1989) Involvement of hormonal and neuromodulatory systems in the regulation of memory storage. Annu Rev Neurosci 12:255-287.

Miller KD (1994) A model of the development of simple cell receptive fields and the ordered arrangement of orientation columns through activity-dependent competition between $\mathrm{ON}$ - and OFF-center inputs. J Neurosci 14:409-441.

Miller KD, MacKay DJC (1994) The role of constraints in Hebbian learning. Neural Comput 6:100-126.

Missal M, Lefevre P, Crommelinck M, Roucoux A (1995) Evidence for high-velocity smooth pursuit in the trained cat. Exp Brain Res 106:509-512.

Miyashita M, Tanaka S (1992) A mathematical model for the selforganization of orientation columns in visual cortex. NeuroReport 3:69-72.

Montague PR, Gally JA, Edelman GM (1991) Spatial signaling in the development and function of neural connections. Cereb Cortex $1: 199-220$

Neuenschwander S, Singer W (1996) Long-range synchronization of oscillatory light responses in the cat retina and lateral geniculate nucleus. Nature 379:728-733.

Olivier E, Grantyn A, Chat M, Berthoz A (1993) The control of slow orienting eye movements by tectoreticulospinal neurons in the cat: behavior, discharge patterns and underlying connections. Exp Brain Res 93:435-449. 
Olson CR, Pettigrew JD (1974) Single units in visual cortex of kittens reared in stroboscopic illumination. Brain Res 70:189-204.

Pettigrew JD (1974) The effect of visual experience on the development of stimulus specificity by kitten cortical neurons. J Physiol (Lond) 237:49-74.

Reid RC, Alonso JM (1995) Specificity of monosynaptic connections from thalamus to visual cortex. Nature 378:281-284.

Robbins TW, Everitt BJ (1995) Arousal systems and attention. In: The cognitive neurosciences (Gazzaniga MS, ed), pp 703-720. Cambridge, MA: MIT.

Rucci M, Tononi G, Edelman GM (1997) Registration of neural maps through value-dependent learning: modeling the alignment of auditory and visual maps in the barn owl's optic tectum. J Neurosci 17:334-352.

Ruderman DL, Bialek W (1994) Statistics of natural images: scaling in the woods. Phys Rev Lett 73:814-817.

Ruthazer ES, Stryker MP (1996) The role of activity in the development of long-range horizontal connections in area 17 of the ferret. J Neurosci 16:7253-7269.

Sejnowski TJ (1977) Storing covariance with nonlinearly interacting neurons. J Math Biol 4:303-321.

Sherk H, Stryker M (1976) Quantitative study of cortical orientation selectivity in visually inexperienced kitten. J Neurophysiol 39:63-70.

Shouval H, Intrator N, Cooper LN (1997) BCM network develops orientation selectivity and ocular dominance in natural scene environment. Vision Res 37:3339-3342.

Sillito AM, Jones HE, Gerstein GL, West DC (1994) Feature-linked synchronization of thalamic relay cell firing induced by feedback from the visual cortex. Nature 369:479-482.

Sindermann F, Geiselmann B, Fischler M (1978) Single motor unit activity in man during fixation and saccades. Electroenceph Clin Neurophysiol 45:64-73.

Singer W (1982) Central core control of developmental plasticity in the kitten visual cortex: I. Diencephalic lesions. Exp Brain Res 47:209-222.

Singer W, Raushecker J (1982) Central-core control of developmental plasticity in the kitten visual cortex II. Electrical activation of mesencephalic and diencephalic projections. Exp Brain Res 47:223-233.

Skavenski AA, Robinson DA, Steinman RM, Timberlake GT (1975) Miniature eye movements of fixation in rhesus monkey. Vision Res 15:1269-1273.
Steinfels GF, Heym J, Strecker RE, Jacobs BL (1983) Behavioral correlates of dopaminergic unit activity in freely moving cats. Brain Res 258:217-228.

Steinman RM, Haddad GM, Skavenski AA, Wyman D (1973) Miniature eye movement. Science 181:810-819.

Stent GS (1973) A physiological mechanism for Hebb's postulate of learning. Proc Natl Acad Sci USA 70:997-1001.

Stryker MP, Harris WA (1986) Binocular impulse blockade prevents formation of ocular dominance columns in the cat's visual cortex. J Neurosci 6:2117-2133.

Toyama K, Komatsu Y, Shibuki K (1984) Integration of retinal and motor signals of eye movements in striate cortex cells of the alert cat. J Neurophysiol 51:649-665.

Trotter Y, Gary-Bobo E, Buisseret P (1981) Recovery of orientation selectivity in kitten primary visual cortex is slowed down by bilateral sections of ophthalmic trigeminal afferents. Dev Brain Res 1:450-454.

Troyer TW, Krukowski AE, Priebe NJ, Miller KD (1998) Contrastinvariant orientation tuning in cat visual cortex: thalamocortical input tuning and correlation-based intracortical connectivity. J Neurosci 18:5908-5927.

van der Schaaf A, van Hateren JH (1996) Modeling the power spectra of natural images: statistics and information. Vision Res 36:2759-2770.

van Hateren JH, van der Schaaf A (1998) Independent component filters of natural images compared with simple cells in primary visual cortex. Proc R Soc Lond B Biol Sci 265:359-366.

Vardi N, Smith RG (1996) The AII amacrine network: coupling can increase correlated activity. Vision Res 36:3743-3757.

Weliki M, Katz LC (1997) Disruption of orientation tuning in visual cortex by artificially correlated neuronal activity. Science 386:680-685.

Wiesel TN, Hubel D (1974) Ordered arrangement of orientation columns in monkeys lacking visual experience. J Comp Neurol 158:307-318

Wilson JR, Sherman SM (1976) Receptive-field characteristics of neurons in the cat striate cortex: changes with visual field eccentricity. J Neurophysiol 39:512-531.

Zahs KR, Stryker MP (1988) Segregation of ON and OFF afferents to ferret visual cortex. J Neurophysiol 59:1410-1429. 